\title{
Application of Improved Empirical Mode Decomposition in Defect Detection Using Vibro-Ultrasonic Modulation Excitation-Fiber Bragg Grating Sensing
}

\author{
Xiao Huang ${ }^{1, \mathrm{a})}$ and Yuegang Tan ${ }^{1, \mathrm{~b}} \mathrm{Li} \mathrm{Cai}^{1, \mathrm{c}}$ \\ 1School of Mechanical and Electrical Engineering, Wuhan University of Technology, 122 Luoshi \\ Road, Wuhan, Hubei, P.R.China \\ a51277619@qq.com , bygtan@whut.edu.cn, ccaili@whut.edu.cn
}

Keywords: Empirical mode decomposition, vibro-ultrasonic modulation, fiber Bragg grating Abstract. The advantage of nonlinear ultrasonic method in micro defect detection has been widely recognized. It has higher sensitivity than linear ultrasonic. Vibro-ultrasonic modulation(VUM) method was adopted in the paper, and fiber Bragg grating(FBG) was used to sense nonlinear signal instead of piezoelectric patches innovatively because it is very easy for FBG to realize distributed measurement. Empirical mode decomposition(EMD) algorithm was improved and applied in the analysis of nonlinear signal. The results shows sidebands signal can be extracted successfully by the improved EMD algorithm.

\section{Introduction}

The evaluation of fatigue damage in kinds of mechanical structures is required for safety. One of the most powerful nondestructive testing(NDT) method is ultrasonic method. Linear ultrasonic NDT has been widely used in the detection of gross defects, whereas it can not work for micro- defect. Hikata [1] first discovered the nonlinear phenomenon when ultrasonic was propagated in a damaged Aluminum structure. Then, many further researchers have proved the existence of defect will lead to nonlinear phenomenon and some acoustic signal whose frequency differs from that of input signal will be produced[2-6]. An important method called vibro-ultrasonic modulation(VUM) excitation method[5]is using vibration to motivate the nonlinear phenomenon.

In order to find out the location of the damage, distributed measurement is inevitable. The common method is to use many piezoelectric patches which are located in different positions. Fiber Bragg grating(FBG), as a new device of optical fiber, is being developed quickly in sensing field. FBG has the electromagnetic interference immunity and it is easier and cheaper than piezoelectric patches to realize distributed measurement. Therefore we used FBG to sense nonlinear response signal instead of piezoelectric patches.

EMD is an important method for the nonlinear signal analysis. It can decompose signal into several $I M F$ s. However, there will be some specific problems in application of EMD in nonlinear ultrasonic signal analysis, some improvement should be made. So in the paper, a system combined VUM excitation and FBG sensing was built, amd EMD algorithm is introduced and improved to analyze the real nonlinear signal.

\section{VUM excitation and FBG sensing system}

\section{Principle of VUM}

In VUM, two inputs are needed, one is vibration with low frequency and the other is ultrasonic with high frequency. When two excitations act on the structure, they can be represented as

$$
s=s_{1}+s_{2}=A_{1} \sin \left(\omega_{1} t+\varphi_{1}\right)+A_{2} \sin \left(\omega_{2} t+\varphi_{2}\right)
$$

where $A_{1}, \omega_{1}, \varphi_{1}$ and $A_{2}, \omega_{2}, \varphi_{2}$ are the amplitude, angular frequency and phase of vibration signal and ultrasonic signal respectively. 
Just considering the elastic material.. If the structure is damaged, there will be nonlinear phenomenon, and the response can be approximately described by

$$
y \approx \alpha s+\beta s^{2}
$$

where $\alpha$ and $\beta$ is linearity constant and one-order nonlinearity coefficient respectively.

$$
\begin{aligned}
& y \approx \alpha A_{1} \sin \left(\omega_{1} t+\varphi_{1}\right)+\alpha A_{2} \sin \left(\omega_{2} t+\varphi_{2}\right)-\beta \frac{A_{1}^{2}}{2} \cos \left(2 \omega_{1} t+2 \varphi_{1}\right)-\beta \frac{A_{2}^{2}}{2} \cos \left(2 \omega_{2} t+2 \varphi_{2}\right) \\
& +\beta A_{1} A_{2} \cos \left[\left(\omega_{2}-\omega_{1}\right) t+\left(\varphi_{2}-\varphi_{1}\right)\right]-\beta A_{1} A_{2} \cos \left[\left(\omega_{2}+\omega_{1}\right) t+\left(\varphi_{2}+\varphi_{1}\right)\right]
\end{aligned}
$$

We can find some components whose frequencies are different from the excitation frequencis will be generated. And the components at $\omega_{2} \pm \omega_{1}$ are called sideband signals. Sideband signals are very important. Firstly, they can be used to judge whether the structure is intact or damaged. Secondly, they can be used to judge the rough size of the defect[5].

Principle of FBG sensing

FBGs are obtained by creating periodic variations in the refractive index of the core of an optical fibre. When broadband light source is input into a FBG, only one wavelength (called Bragg wavelength $\left.\lambda_{B}\right)$ is reflected and others transmitted [7].

$$
\lambda_{B}=2 n_{\text {eff }} \Lambda
$$

where $\Lambda$ is the gratings period, and $n_{\text {eff }}$ is its effective refractive index.

If FBG is under strain caused by vibration and ultrasonic, $\lambda_{B}$ will be changed.

$$
\Delta \lambda_{B} / \lambda_{B 0}=\left(1-P_{e}\right) \varepsilon
$$

where $\lambda_{\mathrm{B} 0}$ is initial wavelength, $\Delta \lambda_{\mathrm{B}}$ is wavelength variation, $P_{e}$ is elasto-optical coefficient of optical fiber, and $\varepsilon$ is the stress caused by vibration and ultrasonic.

\section{VUM excitation-FBG sensing system}

Vibration and ultrasonic were produced by piezoelectric patches. In order to motivate the nonlinearity of defect better, the amplitude of vibration should be large and the best choice of vibration frequency is one of the natural frequency of the structure.

In the FBG sensing system, the optical signal with wavelength information should be transformed into electric signal. In this paper, we use tunable laser light source, optical splitter and optical detector to transform optical signal into electric signal. Fig.1. shows the system block diagram.

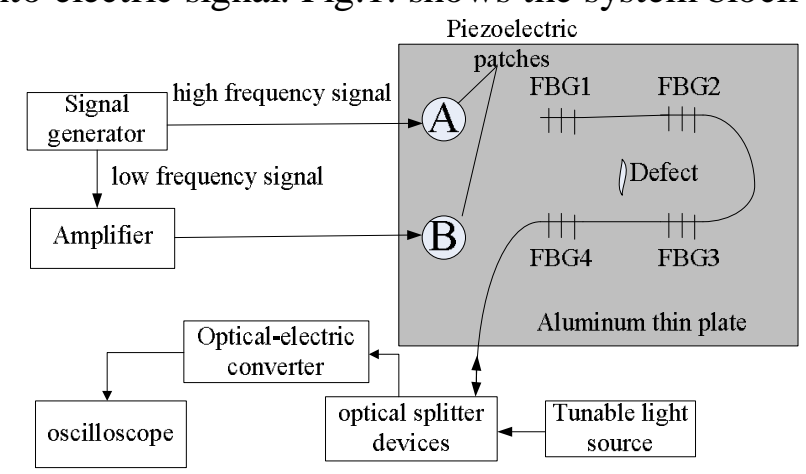

Fig.1. VUM excitation-FBG sensing system block diagram

\section{Improved EMD algorithm}

EMD can be realized according to the following steps[8]:

Step 1.Fix the threshold $\varepsilon$, set $i=1$;.let $r_{0}(t)=x(t)$, 
Step 2. Extract the ith $I M F$ (intrinsic mode function) $c_{i}(t)$ :

(a) Let $h_{0}(t)=r_{i}(t), k=1$,

(b) Extract local maxima and minima of $h_{k-1}(t)$,

(c) Compute upper and lower envelopes $u_{k-1}(t)$ and $v_{k-1}(t)$ using cubic spline interpolating local maxima and minima of $h_{k-1}(t)$ respectively,

(d) Compute the mean of the envelopes: $m_{k-1}(t)=\left(u_{k-1}(t)+v_{k-1}(t)\right) / 2$,

(e) Compute $h_{k}(t)=h_{k-1}(t)-m_{k-1}(t), i=i+1$,

(f) Calculate the following stopping criterion:

$$
S D_{k}=\frac{\sum_{t=0}^{T}\left|h_{k-1}(t)-h_{k}(t)\right|^{2}}{\sum_{t=0}^{T} h_{k-1}^{2}(t)}
$$

(g) Repeat Steps (b)-(f) until $S D_{k}<\varepsilon$ and then put $c_{i}(t)=h_{k}(t)$,

Step 3. Update residual: $r_{i}(t)=r_{i-1}(t)-c_{i}(t)$,

Step 4. Repeat Step 2 with $i=i+1$ until the number of extrema in $r_{i}(t)$ is less than 2;

After EMD, $x(t)$ can be represented by:

$$
x(t)=\sum_{i=1}^{n} c_{i}(t)+r_{n}(t)
$$

Consider the simulated signal:

$$
x_{1}(t)=\cos (4 \pi t)+[2+\cos (0.5 \pi t)] \cos (10 \pi t)+\cos [(10+t) t]
$$

The $I M F$ s of $x_{1}(t)$ is shown in Fig.2. We can find that $I M F_{1}$ is the FM signal of $x_{1}(t), I M F_{2}$ is the AM signal of $x_{1}(t)$, and $I M F_{3}$ is the $\cos (4 \pi \mathrm{t})$. So EMD can decompose the FM and AM signal efficiently.

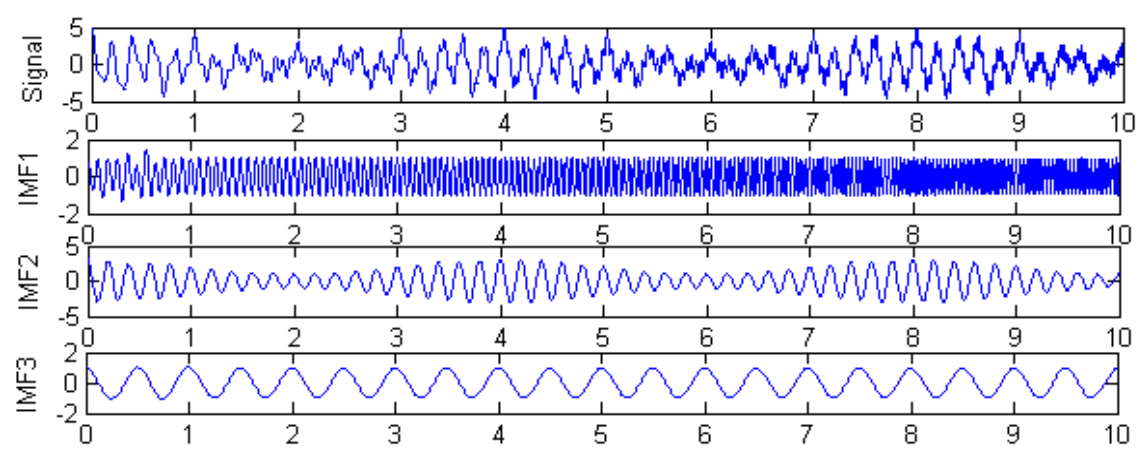

Fig.2. EMD of simulated signal $x_{l}(t)$

Consider another simulated signal: $x_{2}(t)=\sin (8 \pi t)+\sin (10 \pi t)$

Fig.3. and Fig.4. shows the EMD and frequency spectrum of $I M F_{1}$ of simulated signal $x_{2}(t)$. IMF1 is closed to the original signal, in other words, EMD can not decompose the two compositions with different frequencies. Through a lot of experiments and analysis, it is concluded that if the quotient of two frequencies $f_{1} / f_{2}>0.5\left(f_{1}<f_{2}\right)$,EMD can not work because of mode confusion.

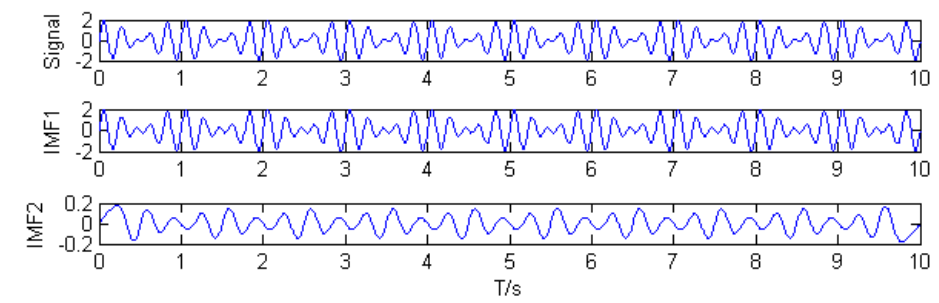

Fig.3. EMD of simulated signal $x_{2}(t)$ 


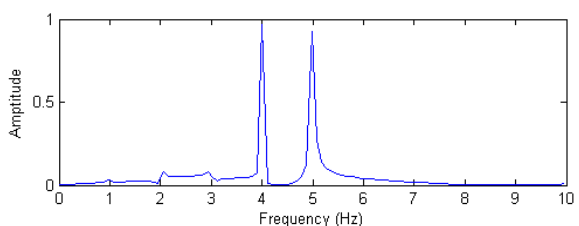

Fig.4. Frequency spectrum(FS) of $I M F_{1}$ of simulated signal $x_{2}(t)$

If we want to decompose such signals, we should improve the EMD algorithm.

Step 1. make EMD, select $I M F$ which includes the main information,

Step 2. select frequency $f_{0}$ satisfying $\left(f_{1}-f_{0}\right) /\left(f_{2}-f_{0}\right)<0.5$,

Step 3. translate the frequency of $x_{2}(t) f_{0}$ to the left,

Step 4. make EMD again,

Step 5. translate the frequency of every $I M F \mathrm{~s} f_{0}$ to the right.

After frequency translate of $x_{2}(t)\left(f_{0}=3.5 \mathrm{~Hz}\right)$, the signal can be represented by

$$
x_{2}{ }^{\prime}(t)=\sin (\pi t)+\sin (3 \pi t)
$$
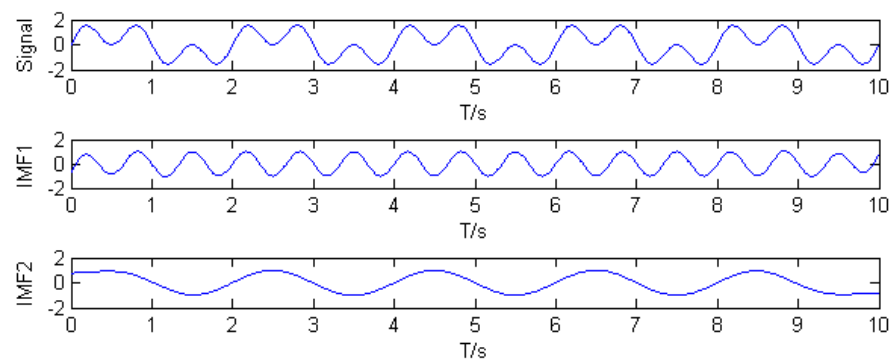

Fig.5. EMD of simulated signal $x^{\prime}{ }_{2}(t)$

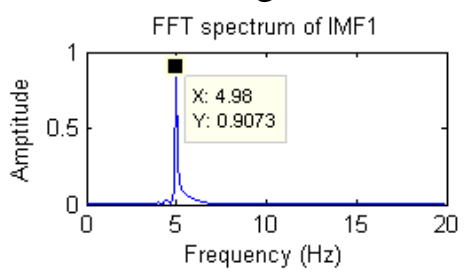

(a) FS of $I M F_{1}$

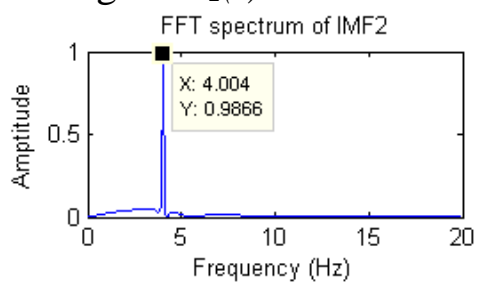

(b) FS of $I M F_{2}$

Fig.6. FS of $I M F$ s of simulated signal $x_{2}{ }_{2}(t)$

From Fig.5. and Fig.6., we can see EMD can work normally after being improved.

\section{Application of improved EMD in nonlinear ultrasonic response}

The nonlinear ultrasonic response was obtained by the VUM excitation-FBG sensing system introduced in Section 2. The frequency of vibration $f_{1}$ and ultrasonic $f_{2}$ were $4 \mathrm{kHz}$ and $62 \mathrm{kHz}$ respectively. We can see that EMD can not decompose the sideband signal from Fig.7 and Fig.8. The improved EMD algorithm was adopted shown in Fig.9-11. IMF 1 in the first and second EMD were both noise.

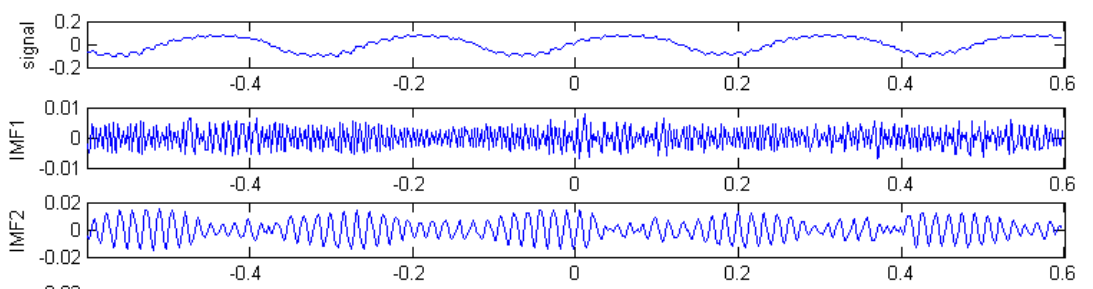

Fig.7. IMFs of response of VUM excitation-FBG sensing system 


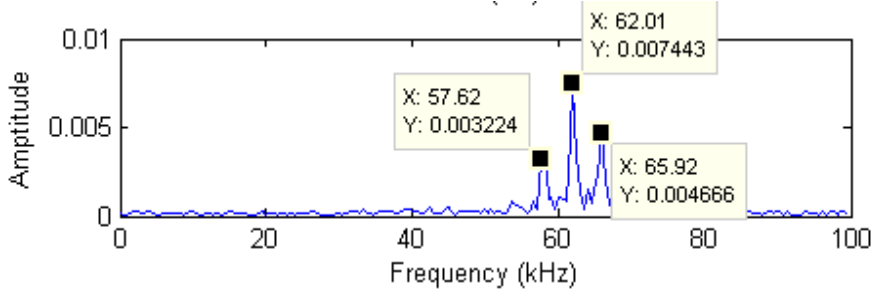

Fig. 8. FS of $I M F_{2}$ of response

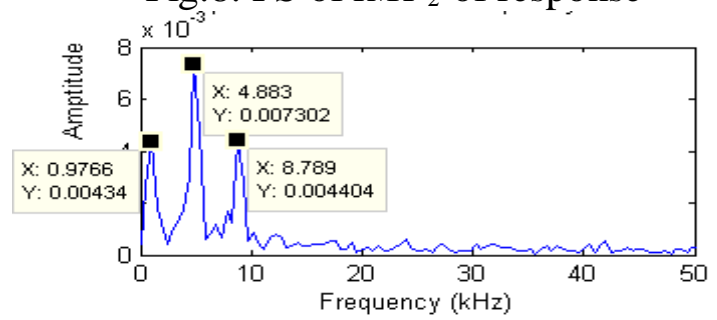

Fig.9. FS of $I M F_{2}$ of response after frequency translation $57 \mathrm{kHz}$ to the left

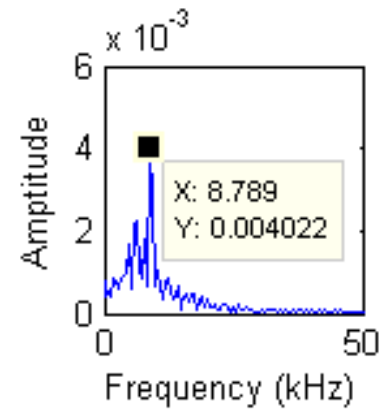

(a) FS of $I M F 2$

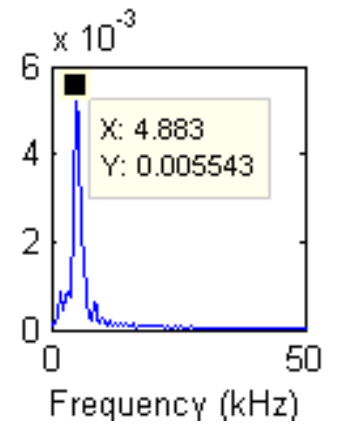

(b) FS of $I M F 3$

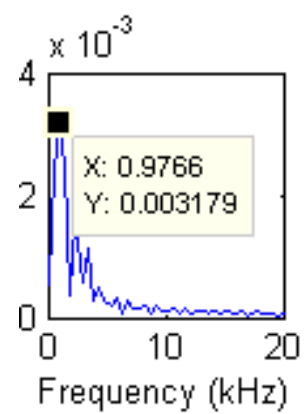

(c) FS of IMF4

Fig.10. Frequency spectrum(FS) of $I M F$ s after second EMD

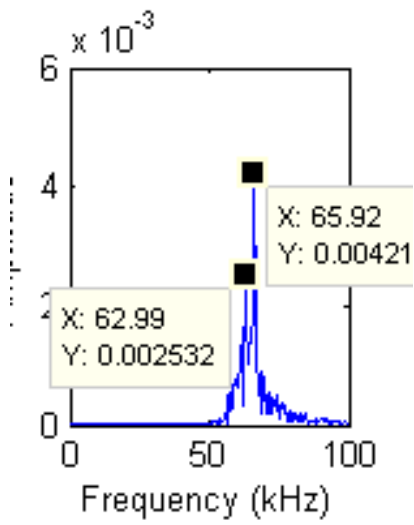

(a) FS of IMF2

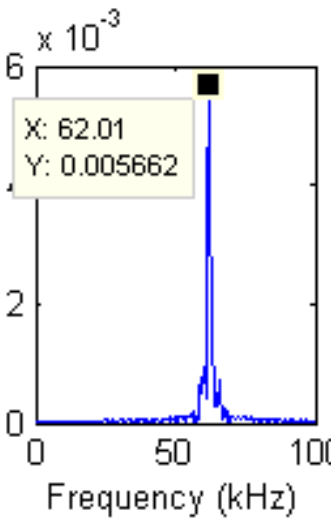

(b) FS of IMF3

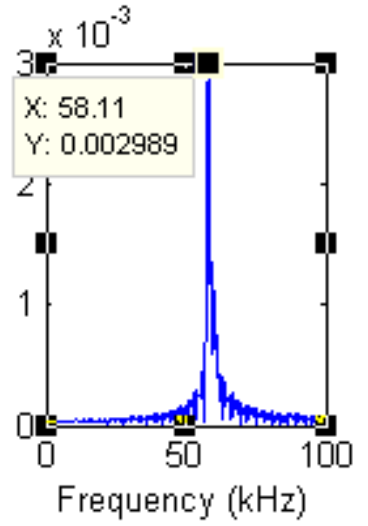

(c) FS of IMF4

Fig.11. FS of $I M F$ s in Fig.14 after frequency translation $57 \mathrm{kHz}$ to the right

We can see from Fig.11. that sideband composition at $58 \mathrm{kHz}\left(f_{2}-f_{1}\right)$ and $62 \mathrm{kHz}\left(f_{2}\right)$ had been decomposed successfully, but composition at $65.9 \mathrm{kHz}\left(f_{2}+f_{1}\right)$ and $62 \mathrm{kHz}$ still had the mode confusion phenomenon, because after translating the frequency $57 \mathrm{kHz}\left(f_{0}\right)$ to the left, $\left(f_{2}\right) /\left(f_{2}+f_{1}\right)=(62-57) /(65.9-57)>0.5$. So choose another frequency $f_{0}$ ' and repeat the improved EMD again can solve the problem.

\section{Conclusions}

This work focused on the application of nonlinear ultrasonic and distributed FBG sensing technique, which has been proven to be a powerful method for NDT. The structure of VUM 
excitation-distributed FBG sensing system was researched and built. In view of the present situation that original EMD algorithm can not decompose the sidebands signal from response; this paper presented an improved EMD algorithm using a frequency translation. And its feasibility was verified by decomposing simulated signal and real nonlinear ultrasonic signal.

\section{Acknowledgements}

This paper was funded and supported by the National Natural Science Foundation of China(51375358).

\section{References}

[1] Hikata A, Chick B B, Elbaum C: "Effect of dislocations on finite amplitude ultrasonic waves in aluminum,". Appl Phys Let 3(1963) 195.

[2] V. V. Kazakov, A. M. Sutin: "Pulsed Sounding of Cracks with the Use of the Modulation of Ultrasound by Vibrations," Acoustical Physics 47(2001) 308.

[3] D. Donskoy, A. Sutin, A. Ekimov: "Nonlinear acoustic interaction on contact interfaces and its use for nondestructive testing," NDT\&E International 34(2001) 231.

[4] Bart Van Damme · Koen Van Den Abeele: "The Application of Nonlinear Reverberation Spectroscopy for the Detection of Localized Fatigue Damage," Nondestruct Eval 33(2014) 263.

[5] HU Haifeng: "Research on Theory and Key Technologies of Nonlinear Ultrasonics for Health Monitoring of Plate-like Metallic Structures,". Ph.D Dissertation, National University of Defense Technology, Changsha (2011).

[6] Charles R. P. Countney, Bruce W. Drinkwater, Simon A. Neild, Paul D.Wilcox: "Factors affecting the ultrasonic intermodulation crack detection technique using bispectral analysis," NDT\&E international 41(2008) 223.

[7] WU Zhaoxia, WU Fei: Principle and Application of FBG Sensing (National Defense Industry Press, Beijing,2011) 28.

[8] Huang, N.E., Shen, Z., Long, S.R., et al: "The empirical mode decomposition and the Hilbert spectrum for nonlinear and non-stationary time series analysis,". Proceedings of the Royal Society of London (1998) 903. 